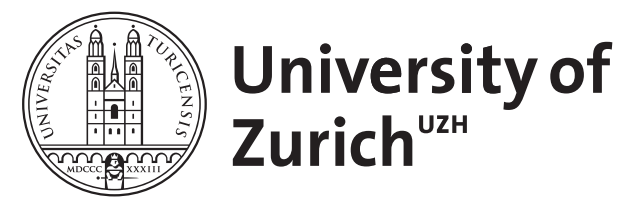

\title{
Psychotherapeutisches Arbeiten in Palliative Care
}

\author{
Gramm, Jan ; Trachsel, Manuel ; Berthold, Daniel
}

\begin{abstract}
In Palliative Care arbeiten multiprofessionell zusammengesetzte Teams für die Verbesserung oder Erhaltung der Lebensqualität von schwerstkranken Patienten und ihren Familien. Klinischen Psychologen und approbierten Psychotherapeuten kommt in diesem Feld ein vielfältiges Spektrum von Aufgaben zu, das von der präventiven und supportiven Arbeit bis hin zum psychotherapeutischen Wirken im engeren Sinne reicht. Auch wenn die Palliativversorgung bislang vielerorts noch keine bedarfsgerechte Begleitung durch Psychologen und Psychotherapeuten leistet, sind entsprechende Entwicklungen deutlich festzustellen. Ziel des vorliegenden Beitrags ist es, einen Überblick über Palliative Care als Anwendungsfeld psychologischer und psychotherapeutischer Interventionen zu geben. Nach einer Skizzierung der relevanten Grundzüge von Palliative Care erfolgt eine Darstellung psychologischer und psychotherapeutischer Themen, subdiagnostischer Befunde sowie psychischer Störungen in der Palliativversorgung. Schließlich soll eine Übersicht psychologischer und psychotherapeutischer Interventionen in Palliative Care gegeben werden.
\end{abstract}

DOI: https://doi.org/10.1159/000504455

Posted at the Zurich Open Repository and Archive, University of Zurich ZORA URL: https://doi.org/10.5167/uzh-179659

Journal Article

Published Version

Originally published at:

Gramm, Jan; Trachsel, Manuel; Berthold, Daniel (2020). Psychotherapeutisches Arbeiten in Palliative Care. Verhaltenstherapie, 30:323-333.

DOI: https://doi.org/10.1159/000504455 


\title{
Psychotherapeutisches Arbeiten in Palliative Care
}

\author{
Jan Gramm ${ }^{\mathrm{a}} \quad$ Manuel Trachsel $^{\mathrm{b}} \quad$ Daniel Berthold ${ }^{\mathrm{a}}$ \\ ${ }^{a}$ Institut für Palliativpsychologie, Friedberg, Deutschland; ${ }^{b}$ Institut für Biomedizinische Ethik und \\ Medizingeschichte, Universität Zürich, Zürich, Schweiz
}

\section{Schlüsselwörter}

Palliative Care · Lebensende · Unheilbar erkrankte

Patienten · Psychologische Intervention · Psychotherapie

\section{Zusammenfassung}

In Palliative Care arbeiten multiprofessionell zusammengesetzte Teams für die Verbesserung oder Erhaltung der Lebensqualität von schwerstkranken Patienten und ihren Familien. Klinischen Psychologen und approbierten Psychotherapeuten kommt in diesem Feld ein vielfältiges Spektrum von Aufgaben zu, das von der präventiven und supportiven Arbeit bis hin zum psychotherapeutischen Wirken im engeren Sinne reicht. Auch wenn die Palliativversorgung bislang vielerorts noch keine bedarfsgerechte Begleitung durch Psychologen und Psychotherapeuten leistet, sind entsprechende Entwicklungen deutlich festzustellen. Ziel des vorliegenden Beitrags ist es, einen Überblick über Palliative Care als Anwendungsfeld psychologischer und psychotherapeutischer Interventionen zu geben. Nach einer Skizzierung der relevanten Grundzüge von Palliative Care erfolgt eine Darstellung psychologischer und psychotherapeutischer Themen, subdiagnostischer Befunde sowie psychischer Störungen in der Palliativversorgung. Schließlich soll eine Übersicht psychologischer und psychotherapeutischer Interventionen in Palliative Care gegeben werden.

c 2019 S. Karger AG, Basel
Psychotherapeutic Work in Palliative Care

\section{Keywords}

Palliative care - End of life - Terminally ill patients . Psychological intervention · Psychotherapy

\section{Abstract}

In palliative care, multi-professional teams work to improve and maintain the quality of life of terminally ill patients and their families. In this field, clinical psychologists and psychotherapists take on a broad variety of tasks that range from preventive and supportive to psychotherapeutic work. Even if the provision of palliative care frequently does not yet offer appropriate need-based care by psychologists and psychotherapists, developments to that effect are clearly noticeable. The aim of the present article is to give an overview of palliative care as an area of application for psychological and psychotherapeutic interventions. After depicting relevant features of palliative care, characteristic topics, strains and mental disorders in palliative care will be briefly delineated. Finally, an overview of psychological and psychotherapeutic interventions for palliative care will be presented.

c) 2019 S. Karger AG, Basel

\section{KARGER}

(C) 2019 S. Karger AG, Basel
Dr. rer. med. Dipl.-Psych. Daniel Berthold 
Ein reicher Kaufmann in Bagdad schickte eines Tages seinen Diener mit einem Auftrag zum Basar. Als dieser zurückkam, zitterte er vor Angst am ganzen Leibe. "Herr", rief er, "auf dem Markt sah ich einen Fremden. Ich blickte ihm ins Gesicht und erkannte, dass es der Tod war! Er hob die Hand drohend gegen mich und ging davon. Nun habe ich große Angst. Bitte, Herr, gebt mir ein Pferd, damit ich sofort nach Samarra reiten kann, um möglichst weit fort vor dem Tod zu fliehen.” Der Kaufmann war sehr besorgt um seinen treuen Diener und gab ihm sein schnellstes Pferd. Der Diener schwang sich auf den Rücken des Tieres und raste im wilden Galopp davon. Später am Tag ging der Kaufmann selbst zum Basar, um noch etwas zu erledigen. Dort sah er den Tod in der Menge der Menschen. Er ging auf ihn zu und fragte: "Du hast heute morgen meinem Diener gedroht. Was hatte das zu bedeuten?" "Ich habe dem Mann keineswegs gedroht. Meine Geste drückte viel mehr mein Erstaunen aus, ihn hier anzutreffen. Mir hatte man nämlich gesagt, dass ich ihn heute Abend in Samarra treffen würde."

William Somerset Maugham [1933]

\section{Einleitung}

Diese Parabel Maughams mag das Verhältnis der modernen Medizin zum Tod treffend beschreiben: Obwohl chronisch fortschreitende Erkrankungen seit jeher mit Komorbidität sowie einer stetigen Verschlechterung des Allgemeinzustandes einhergehen und viele dieser Erkrankungen letztlich in den Tod münden, wird der Blick nicht auf das Sterben, sondern auf vermeintliche Heilungsmöglichkeiten gerichtet. Diese Vermeidungsstrategie zieht letztlich nutzlose Behandlungen (medical futility) nach sich [Schneiderman et al., 1990; Meran, 2003; Becker und Blum, 2004]. In der Folge trifft der Tod nicht nur Patienten und Angehörige, sondern auch die Behandler weitgehend unvorbereitet.

Indem Palliative Care kritische Themen im Kontext unheilbarer Erkrankungsverläufe nicht tabuisiert, sondern explizit benennt, haben Patienten und Angehörige die Möglichkeit, sich auf die noch verbleibende Zeit und die Umstände des Lebensendes einzustellen. Vor allem wird es aber für das Palliative Care-Team möglich, Therapieziele zu formulieren, auf die in Anbetracht der medizinischen Situation (Indikation), nach Maßgabe des Patientenwillens und gegebenenfalls unter Berücksichtigung der Angehörigenbedürfnisse, hingearbeitet werden kann. Im Unterschied zu einer somatisch-defizitorientierten Medizin werden Therapieziele also nicht alleine im Hinblick auf das Krankheitsgeschehen definiert. Vielmehr findet eine Kontextualisierung statt, mit der Konsequenz, dass neben der Effektivität immer auch die Frage nach der Sinnhaftigkeit einer Behandlung ins Blickfeld rückt [Sauer und Weiske, im Druck], z.B. wenn die Ermittlung des mutmaßlichen Willens ergibt, der komatöse Patient habe eine künstliche Ernährung immer abgelehnt.
Über die Frage nach der Sinnhaftigkeit werden veränderungsorientierte Therapieziele durch solche ergänzt, die ein Akzeptieren des Unveränderlichen nicht als Misserfolg, sondern als realistische Option beinhalten. Letztlich brachte die Palliativbewegung damit in die Medizin, was über die dritte Welle der Verhaltenstherapie Einzug in die psychotherapeutische Versorgung hielt [Heidenreich et al., 2007; Heidenreich und Michalak, 2013].

Psychologen und Psychotherapeuten in Palliative Care kommt ein äußerst vielfältiges Spektrum an Aufgaben zu, wie etwa in der Teambegleitung, Lehre und Fortbildung, Forschung sowie in der Öffentlichkeitsarbeit [Berufsverband Deutscher Psychologinnen und Psychologen (BDP), 2016]. Der vorliegende Beitrag fokussiert auf die klinisch-psychologische Arbeit mit Patienten und ihren Angehörigen.

\section{Rahmenbedingungen psychologischen und psychotherapeutischen Arbeitens}

\subsection{Palliative Care als multiprofessionelles Arbeitsfeld}

Die Weltgesundheitsorganisation versteht unter Palliative Care einen Ansatz, "der die Lebensqualität von $\mathrm{Pa}$ tienten und deren Familien verbessert, die mit den Problemen im Zusammenhang einer lebensbedrohenden Erkrankung konfrontiert sind, dies mittels Prävention und Linderung von Leiden durch frühzeitiges Erkennen und umfassende Erfassung sowie durch die Behandlung von Schmerz und anderen Problemen, auf körperlicher, psychosozialer und spiritueller Ebene" [World Health Organization (WHO), 2002; Übersetzung durch die Deutsche Gesellschaft für Palliativmedizin (DGP), 2016]. Aus dieser Definition lässt sich eine Reihe von Implikationen ableiten, die das Selbstverständnis von Palliative Care charakterisieren: Zentral ist der Anspruch einer bio-psychosozio-spirituellen Behandlung zu nennen, die sich an die Notwendigkeit eines multiprofessionellen Versorgungssettings knüpft. Mit der Lebensqualität als vorrangigem Therapieziel erfährt das subjektive Erleben einen besonderen Stellenwert: "Pain is what the patient says it is." Die Angehörigen explizit als (Mit-)Betroffene zu benennen, unterstreicht die genuin systemische Sichtweise des Arbeitsfeldes: Patienten werden nicht als unabhängige Individuen verstanden, sondern immer als eingebunden in wechselwirkende Systeme aus Familie und Behandler.

\subsection{Das Berufsbild der Palliativpsychologie}

Trotz des Anspruchs einer multiprofessionellen Versorgung ist die Einbindung von Psychologen und Psychotherapeuten in die palliativen und hospizlichen Versorgungsstrukturen bislang nicht klar geregelt. Daher gibt es auch keine eindeutigen gesetzlichen Vorgaben be- 
züglich der Qualifizierung. Von der Sektion Psychologie der DGP wurde das Berufsbild der "Palliativpsychologie" erarbeitet, das vom BDP in den Katalog der psychologischen Berufsbilder aufgenommen wurde [Sektion Psychologie der DGP, 2016; BDP, 2016]. Das Berufsbild führt auf, was Psychologen in Palliative Care leisten - unabhängig davon, ob diese über eine psychotherapeutische Zusatzqualifikation beziehungsweise eine Approbation verfügen oder nicht. Demnach ist die Palliativpsychologie ein Teilgebiet von Gesundheitspsychologie und klinischer Psychologie, wobei die Arbeit mit Patienten und Angehörigen zwar eine wichtige, aber nicht die einzige Tätigkeit von Psychologen in Palliative Care darstellt [Münch et al., 2016].

\subsection{Das palliative Patientenklientel}

Palliative Patienten unterscheiden sich in vielfacher Hinsicht vom "klassischen" Patientenklientel der psychotherapeutischen Versorgung. Der Erstkontakt erfolgt zumeist im Rahmen einer vordergründig palliativen oder hospizlichen Versorgungssituation, d.h. bei Vorliegen einer lebenslimitierenden fortschreitenden Erkrankung. Hierzu zählen neben Krebserkrankungen kardiovaskuläre Erkrankungen (z.B. Herzinsuffizienz), Erkrankung von Nieren oder Leber, pulmonale Erkrankungen (z.B. COPD), neurologische Erkrankungen (z.B. amyotrophe Lateralsklerose, Demenz), Stoffwechselerkrankungen (z.B. Diabetes mellitus), Infektionskrankheiten (z.B. AIDS) oder altersbedingte Multimorbidität ("FrailtySyndrom").

Psychologen und Psychotherapeuten, die in die Behandlung körperlich schwerkranker Patienten eingebunden sind, benötigen daher Wissen über spezifische Krankheitsverläufe und die damit verbundenen Herausforderungen bezüglich Prognose und Behandlung. So besteht etwa ein großer Unterschied zwischen dem zumeist stetigen Verlauf einer Krebserkrankung im Endstadium und dem typischerweise stark oszillierenden Verlauf einer Herzerkrankung, bei der nur schwer einschätzbar ist, ob sich ein Patient von einer Krise wieder erholen wird oder nun der Punkt erreicht ist, sich auf das Sterben einzustellen [Bausewein, 2005]. In palliativen Situationen häufig auftretende körperliche Symptome, über die Psychologen und Psychotherapeuten unbedingt Kenntnis haben sollten, sind etwa Schmerzen, Schwäche, Dyspnoe, Übelkeit, Verstopfung, Fatigue, Schlafstörungen, Verwirrtheit und Appetitlosigkeit [siehe etwa Trachsel und Maercker, 2016].

\subsection{Das palliative Setting}

Palliative Erkrankungsverläufe gehen mit einer hohen Vulnerabilität der Patienten einher. Für die psychologische und psychotherapeutische Arbeit mit Sterbenden bedeutet dies, dass bestimmte Voraussetzungen beachtet werden müssen, die sich durch folgende Aspekte charakterisieren lassen:

- Unvorhersehbar wechselhafte Tagesform des Patienten, sodass anstelle der üblichen 50-Minuten-Sitzung eine deutlich flexiblere Handhabung notwendig ist.

- Eingeschränkte oder nicht vorhandene Mobilität, sodass im ambulanten Setting in der Regel nur Hausbesuche möglich sind.

- Eingeschränkte Freiheitsgrade der pflegenden Angehörigen, sodass auch diese nur schwer eine psychotherapeutische Praxis aufsuchen können.

- Oft eingeschränkte Konzentrationsfähigkeit beziehungsweise herabgesetzte Vigilanz, sodass eine hohe Flexibilität in der Terminfindung geboten ist.

- Häufig eingeschränkte Sprechfähigkeit (aufgrund von Schwäche, Dyspnoe oder Aphasie bei zerebralen Metastasen oder bei neurologischen Erkrankungen).

- Begrenzte (Lebens-)Zeit, sodass auch die psychologische und psychotherapeutische Arbeit einem gewissen Zeitdruck unterliegt.

Insbesondere aus dem letztgenannten Punkt ergibt sich, dass eine Sitzung - trotz der genannten Einschränkungen - auch zwei Stunden und länger andauern kann, wenn etwa sowohl mit dem Patienten als auch mit Angehörigen erst separat und anschließend gemeinsam gearbeitet wird. Eine therapeutische Beziehung nach klassischem Verständnis kann aufgrund der begrenzten Zahl an Kontakten meist nicht aufgebaut werden. Stattdessen muss innerhalb kurzer Zeit - oft in den ersten Minuten der ersten und vielleicht einzigen Sitzung - eine hinreichend tiefe Begegnung stattfinden, um die wesentlichen Themen zu identifizieren und bearbeiten zu können.

Die besonderen Umstände des palliativen Settings spiegeln sich in dem Befund wieder, dass eine manualisierte kognitive Verhaltenstherapie bei Palliativpatienten sich als kaum durchführbar herausstellte, da aufgrund der schweren körperlichen Beeinträchtigung nur 31\% der Patienten in der Lage waren, an mindestens acht von insgesamt zwölf Therapiesitzungen teilzunehmen [Serfaty et al., 2019].

\section{Psychologische und psychotherapeutische Themen, subdiagnostische Befunde und psychische Störungen}

\subsection{Psychologische und psychotherapeutische Themen}

3.1.1 Patienten

Neben der körperlichen Symptomatik durchleben Palliativpatienten im Verlauf ihrer Erkrankung oft ein breites Spektrum an psychologischen und psychotherapeutischen Themen, innerhalb derer Gefühle wie Trauer, Niedergeschlagenheit, Verzweiflung, Angst, Furcht, Unsicherheit und Einsamkeit eng beieinander liegen [Block, 2006]. 
$\mathrm{Zu}$ den großen Themen in der Palliativversorgung zählen bevorstehende Entscheidungen, etwa hinsichtlich der Behandlungsoptionen oder der weiteren Versorgung. Ein nicht weniger bedeutsames Thema ist das Sanktionieren von Gefühlen und Kognitionen. Damit einher geht oft die Intention, die emotionale Abwärtsspirale unterbrechen zu wollen, was aber meist den gegenteiligen Effekt auslöst: Die zunehmende Entfremdung der Familienmitglieder aufgrund der zurückgehaltenen Gefühle und Kognitionen. Oft wird auch die Frage nach dem richtigen Umgang mit dem Thema "Sterben" in der Familie gestellt. Darüber hinaus zählen Demoralisierung, Verbitterung, Sinnverlust, Überforderung, Hilflosigkeit, Hoffnungslosigkeit, Autonomieverlust, kognitive Einschränkungen und Konzentrationsstörungen, Schlafstörungen, Stimmungsschwankungen, Veränderungen des Körperbilds, Schuldgefühle, Nutzlosigkeit, Isolationserleben, Würdeverlust, Scham, das Gefühl, nur noch eine Belastung zu sein, und der Wunsch zu Sterben zum häufigen Gegenstand psychologischer und psychotherapeutischer Sitzungen in Palliative Care [Trachsel und Maercker, 2016]. Daneben gibt es aber auch den Bedarf, sich mit Themen auseinanderzusetzen, die von Sterbenden und Angehörigen als ressourcenstärkend erlebt werden, etwa das Ziehen einer Lebensbilanz, das Ausdrücken von Dankbarkeit, das Regeln letzter Dinge, der Wunsch nach Versöhnung oder das Finden inneren Friedens. Eine Meta-Synthese von 35 qualitativen Studien ergab vier Themenfelder, mit denen sich Patienten mit einer terminalen Krebserkrankung konfrontiert sehen: (1) Das Trauma des katastrophalen Einbruchs des bisherigen Lebens (trauma), (2) das Leben als "Krebspatient" mit all den krankheits- und behandlungsbedingten Beeinträchtigungen, zugehörigen Emotionen wie Angst oder Schuld und Auswirkungen auf das soziale Leben (life as a cancer patient), (3) die Erfahrung der vom gesunden Umfeld nicht geteilten Liminalität (liminality) - in der Ethnologie ein Schwellenzustand zwischen einer verlassenen alten und noch nicht erreichten neuen Ordnung - sowie (4) sein Leben trotzdem leben (holding on to life) [Willig und Wirth, 2018].

\subsubsection{Angehörige}

Angehörige schwerstkranker Menschen sind in der Doppelrolle als Hilfegebende und Mitbetroffene häufig hochbelastet. Pflegende Angehörige sind besonders durch die durchgängig erforderliche Anwesenheit, das Miterleben von Leid, durch Übergänge von stationärer zu häuslicher Versorgung und durch körperliche Anforderung belastet [Kern et al., 2009]. Sie sind mit vielen Anforderungen konfrontiert, die psychisch stark belasten können, z.B. Sorge um das Wohl des Patienten, Organisation und Management von Behandlungen und Hilfsangeboten, Zukunftsangst, gestörter Schlaf, Abschiedneh- men, Entscheidungen treffen und Ungewissheit über den weiteren Krankheitsverlauf.

Überdies verändern sich familiäre Rollen, reduzieren sich soziale Kontakte oder stellen sich erhebliche finanzielle Belastungen ein. So kann die Erkrankung in der Familie zum "organisierenden Prinzip" werden [Kröger et al., 2002], etwa wenn sich die Wochenplanung der Familie an den vorgegebenen Behandlungsterminen ausrichten muss. Zu Bedenken ist auch, dass die betroffenen Familien neben einer Krankheitsgeschichte immer auch eine Behandlungsgeschichte aufzuweisen haben, die oft mit würdeverletzenden Erfahrungen verbunden ist.

\subsection{Subdiagnostische Befunde}

Psychische Symptome ohne erfüllte Diagnosekriterien finden sich bei onkologischen und palliativen Patienten wesentlich häufiger als in der Allgemeinbevölkerung [Mitchell et al., 2011]. In diesem Zusammenhang haben sich verschiedene Begriffe etabliert wie subdiagnostisch, subsyndromal, subkategorial, subklinisch oder unterschwellig [Röhrle et al., 2008]. Der Übergang von einer normalen psychischen Reaktion zu einer Reaktion mit Störungswertigkeit ist nicht immer klar abgrenzbar und bedarf einer genauen Differenzierung. Unabhängig davon ist der Bedarf an psychologischer oder psychotherapeutischer Begleitung einzuschätzen: So kann es durchaus notwendig werden, $\mathrm{Pa}$ tienten und Angehörigen psychologischen Support anzubieten, obwohl das gezeigte Verhalten in Anbetracht der palliativen Situation durchaus angemessen erscheinen mag. In Palliative Care ist die Frage der Behandlungswürdigkeit höher zu bewerten als die Frage der Störungswertigkeit.

\section{Beispiel: Demoralisierung}

Die Demoralisierung geht über das Gefühl der Niedergeschlagenheit hinaus, erfüllt aber nicht die Diagnosekriterien einer psychischen Störung. Dennoch bedarf sie einer psychologischen Behandlung. In klinisch relevanter Ausprägung tritt sie bei ca. 13-18\% aller fortgeschrittenen onkologischen Patienten auf [Robinson et al., 2015; Mehnert et al., 2011]. Die Demoralisierung ist gemäß Clarke und Kissane [2002] definiert durch:

- Affektive Symptome existenzieller Belastung wie Hoffnungslosigkeit, Verlust von Sinngebung und Lebensaufgaben.

- Kognitive Einstellungen geprägt durch Pessimismus, Hilflosigkeit, Gefühle, gefangen zu sein, persönliches Versagen und Fehlen einer erstrebenswerten Zukunft.

- Fehlen von willentlichem Antrieb und der Motivation, die Situation anders zu bewältigen.

- Merkmale von sozialer Entfremdung oder Isolation sowie Fehlen von Unterstützung.

- Fluktuation in der emotionalen Intensität.

- Kein Vorliegen einer primären Major Depression oder anderen psychischen Störung. 
Während sich eine Depression wesentlich durch den generellen Verlust von Freude, Genussfähigkeit und Interesse kennzeichnet, ist eine Demoralisierung geprägt durch den Verlust von Sinn und Hoffnung sowie antizipierter Freude, während die Genussfähigkeit für den Moment aber erhalten bleibt [Robinson et al., 2015]. Während die Ursache von Depression nicht immer klar bestimmbar ist, besteht ein deutlicher Zusammenhang zwischen Demoralisierung und körperlicher Symptomlast, durch welche die Aktivität eingeschränkt wird (Fatigue, Immobilität, Dyspnoe, Konzentrationsschwäche). Stark ausgeprägte Demoralisierung korreliert dabei hoch mit Depression, Angst und Sterbewunsch [Mehnert et al., 2011]. Es besteht ein negativer Zusammenhang mit Lebensqualität und Lebenssinn [Robinson et al., 2015]. Im Umkehrschluss ergibt sich hieraus eine besondere Indikation von Interventionen, die zur Unterstützung von Lebensqualität und Lebenssinn beitragen.

\subsection{Psychische Störungen \\ 3.3.1 Patienten}

Sucht man nach Forschungsbefunden zu den Prävalenzen psychischer Störungen, so fällt eine erhebliche Variationsbreite der Statistiken ins Auge [Mitchell et al., 2011]. Grund hierfür ist wohl auch, dass es sich als schwierig erweist, Störungen eindeutig zu identifizieren, insofern nämlich die Symptomatik teilweise auch auf die somatische Erkrankung selbst oder deren Behandlung zurückzuführen ist [Endicott, 1984; Miller und Massie, 2015]. Die psychiatrische Schablone erweist sich aber auch bei psychischen Symptomen nur als bedingt nützlich, so etwa, wenn depressionstypische Symptome bei Palliativpatienten zwar häufig auftreten - und auch behandlungsbedürftig sind - aber eben nicht zwingend als Hinweis auf eine zugrundeliegende Depression zu werten sind. Man sollte demnach Vorsicht walten lassen bei der Frage der Störungswertigkeit und Umsicht walten lassen bei der Frage der Behandlungswürdigkeit.

Als Beispiel sei der bei Palliativpatienten häufig geäußerte Wunsch nach vorzeitigem Sterben (wish to hasten death) genannt, der jedoch meist nicht als Suizidalität und somit auch nicht als Hinweis auf eine Depression verstanden werden kann [Block, 2000; Monforte-Royo et al., 2012]. In der Praxis erweist sich daher eine Differenzierung zwischen Sterbewunsch und Sterbewille als sinnvoll [Ohnsorge et al., 2014].

Liegen aber psychische Symptome mit erfüllten Diagnosekriterien für eine psychische Störung vor, so können diese - in enger Absprache mit den Patienten durchaus auch störungsspezifisch behandelt werden. Palliative Care heißt nicht, dass neben dem palliativen Ansatz keine Interventionen mit kurativer Intention durchgeführt werden sollten, wenn eine solche erfolgversprechend ist [Ferris et al., 2002]. Bei allen Arten psychischer Störungen am Lebensende, wie depressiven Störungen, Angst- oder Zwangsstörungen, kann sowohl eine (psycho-)therapeutische Behandlung gemäß den geltenden Leitlinien (für die Unipolare Depression; siehe z.B. [Leitlinienprogramm Onkologie (Deutsche Krebsgesellschaft, Deutsche Krebshilfe, AWMF), 2015]) oder eine der Situation des Patienten am Lebensende angepasste therapeutische Intervention durchgeführt werden. Für ausgedehnte störungsspezifische Psychotherapien reicht die Zeit bei Patienten am Lebensende oft nicht. Kürzere supportive Ansätze eignen sich hierfür besser. Auch pharmakologisch existieren einige schneller wirksame Optionen. So bietet sich etwa bei einer depressiven Episode am Lebensende, wenn die Dauer von zwei bis sechs Wochen bis zum Wirkungseintritt klassischer Antidepressiva nicht abgewartet werden kann, der Einsatz von Opioiden oder Ketamin an [siehe etwa Andrade, 2017].

Drei Beispiele von Herausforderungen bei Palliativpatienten mit spezifischen psychischen Störungen werden nachfolgend skizziert.

\section{Beispiel 1: Persönlichkeitsstörungen}

Unabhängig davon, ob psychische Symptome mit oder ohne erfüllte Diagnosekriterien für eine psychische Störung vorliegen, sind Professionelle in Palliative Care oft mit Patienten konfrontiert, die eine vorbestehende Persönlichkeitsstörung oder akzentuierte Persönlichkeitszüge vorweisen. $\mathrm{Da}$ in der ambulanten Palliativversorgung und in Hospizen kaum Psychologen oder Psychotherapeuten integriert sind, muss bei solchen Patienten, die oft als "schwierig" wahrgenommen und bezeichnet werden, von einer generellen Unterversorgung ausgegangen werden. Patienten mit Persönlichkeitsstörungen oder akzentuierten Persönlichkeitszügen in der Palliativversorgung gehen daher mit einer erhöhten Belastung der Pflegekräfte einher. So wird beispielsweise ein Patient mit hohem Betreuungsbedarf und einer langsam verlaufenden terminalen Erkrankung, etwa einer amyotrophen Lateralsklerose, der an einer zwanghaften Persönlichkeitsstörung leidet, das Pflegeteam in hohem Maße herausfordern.

\section{Beispiel 2: Anorexia nervosa}

Bei der Anorexia nervosa, die durch einen absichtlich selbst herbeigeführten oder aufrechterhaltenen Gewichtsverlust charakterisiert ist, kann es trotz jahrelanger kompetenter Behandlung inklusive störungsspezifischer Psychotherapie und Zwangsernährung zur Situation kommen, in welcher eine weitere therapeutische Behandlung keinen oder nur noch einen sehr geringen Nutzen 
verspricht. Trachsel et al. [2016] beschreiben die folgende Patientin:

"Die 19-jährige Lisa S. [...] ist aufgrund einer Anorexia nervosa und des dadurch selbst herbeigeführten hochgradigen Gewichtsverlusts so stark mangelernährt, dass aus medizinischer Sicht nicht ausgeschlossen werden kann, dass ihr Leben akut gefährdet ist. Gleichzeitig lehnt die Patientin weitere therapeutische Maßnahmen dezidiert ab und stellt sich damit gegen den Willen ihrer Eltern und gegen die Empfehlung der behandelnden Fachpersonen" [Trachsel et al., 2016, S. 171-173].

Diese Patientin ist somit massiv untergewichtig, hat keinerlei Behandlungsmotivation mehr und steht am Lebensende. Dies ist zweifelsohne eine Situation, bei der von einer palliativen Situation aufgrund einer psychischen Störung gesprochen werden kann, obwohl der Sterbeprozess in diesem Fall prinzipiell reversibel ist und kein expliziter Sterbewunsch vorhanden ist, sondern aufgrund der Körperschema-Störung eine tiefe Überzeugung seitens der Patientin, (immer noch) zu wenig schlank zu sein.

Bei Lisa S. ergeben sich deshalb für die behandelnden Professionellen folgende ethische Herausforderungen:

“Zum einen muss eine Einschätzung der Einwilligungsfähigkeit der Patientin erfolgen und es muss in jedem Fall weitest möglich Respekt vor ihrer Autonomie gewährleistet sein, zum anderen gibt es die moralische Pflicht der Fachpersonen, das Leben der Patientin zu schützen und insbesondere dann einzugreifen, wenn Zweifel an ihrer Einwilligungsfähigkeit bestehen" [Trachsel et al., 2016, S. 173].

Beispiel 3: Psychose oder Delir

Bei vorbestehender Psychose oder vorbestehendem Delir wird eine Abklärung der Einwilligungsfähigkeit notwendig, wenn Zweifel bestehen, ob ein Patient in der Lage ist, sich für oder gegen eine Behandlungsoption zu entscheiden [Trachsel et al., 2014a]. Liegt eine gültige $\mathrm{Pa}$ tientenverfügung vor und hat der Patient im Rahmen seiner Erkrankung seine Einwilligungsfähigkeit hinsichtlich einer bestimmten medizinischen Entscheidung verloren (z.B. bezüglich Zustimmung zu einer vorgeschlagenen Intervention), so tritt die Patientenverfügung in Kraft, sofern deren Inhalt sich auf die aktuelle Situation anwenden lässt. In diesem Fall muss eine gültige Patientenverfügung vom Behandlungsteam zwingend eingehalten werden, wobei jedoch immer ein gewisser Ermessensspielraum aufgrund der verwendeten Formulierungen besteht [Trachsel et al., 2014b].

Eine Patientenverfügung ist jedoch nur dann gültig, wenn der Patient bei der Verfassung derselben in einem Zustand der Einwilligungsfähigkeit war. Falls diese zum Zeitpunkt der Abfassung von keinem Arzt bestätigt wurde, kann es retrospektiv schwierig abzuschätzen sein, ob bei der Verfassung der Patientenverfügung die Einwilligungsfähigkeit tatsächlich gegeben war. Insbesondere bei Patienten mit vorbestehenden Erkrankungen, die oft mit einer aufgehobenen Einwilligungsfähigkeit einher gehen, ist die retrospektive Beurteilung besonders herausfordernd. Dies kann zum Beispiel auf Patienten mit Psychosen oder Delirien in der Vorgeschichte zutreffen. Besteht ein klarer Hinweis, dass ein Patient bei der Verfassung der Patientenverfügung in einem psychotischen Zustand war, kann dieser Umstand als Argument für die Ungültigkeit einer Patientenverfügung herangezogen werden.

\subsubsection{Angehörige}

Das Ausmaß der Morbidität bei pflegenden Angehörigen stellt sich in aktuellen Studien als höher dar als es bisherige Untersuchung ergaben [Grande et al., 2018]. Preisler und Goerling [2016] zeigen auf, dass sich psychische Belastetheit von Angehörigen oft in höherer Ausprägung vorfindet als bei den Patienten selbst. Beschrieben werden hier Angsterkrankungen, Depressionen und Anpassungsstörungen.

\section{Beispiel: Komplizierte Trauer}

Analog wie Niedergeschlagenheit zu Depression verhält sich Trauer zu komplizierter Trauer. Obwohl Trauer eine natürliche Reaktion auf eine Verlusterfahrung darstellt, können sich schwierige Trauerverläufe und eine komplizierte Trauer entwickeln. Komplizierte Trauer stellt eine eigenständige psychische Entität dar [Prigerson et al., 1996; Znoj, 2016], weshalb die WHO in der Neufassung der ICD hierfür folgerichtig eine spezifische Diagnose ("Prolonged Grief Disorder") vorsieht. Im Gegensatz zu einer depressiven Episode zeigt sich die Stimmungslage bei Trauer fluktuierend. Freude kann sowohl im Moment als auch auf bestimmte Ereignisse hin empfunden werden, das Selbstwertgefühl ist intakt, der Wunsch nach vorzeitigem Sterben kann auftreten (nicht jedoch Suizidalität) [Block, 2006; Strada, 2009]. Eine gründliche Differenzialdiagnostik hat direkte Auswirkungen auf die Therapie, denn eine störungswertige Trauer kann nicht wie eine Depression behandelt werden. Sie erfordert spezifisch entwickelte Interventionen wie etwa die Family Focused Grief Therapy [Kissane et al., 2006]. In Abgrenzung zur Depression ist der Einsatz von Antidepressiva weder bei der Trauer noch bei der Demoralisierung angezeigt.

Es sei erwähnt, dass das Auftreten von Trauer nicht nur nach dem Tod, sondern bereits in der palliativen Betreuungsphase diskutiert wird, und zwar sowohl aufseiten der Angehörigen ("antizipierte Trauer") wie auch aufseiten der Patienten selbst ("preparatory grief") [Strada, 2009]. Antizipierte Trauer kann sich ähnlich äußern wie Trauer nach einem Verlust, beispielsweise durch Trennungsschmerz, Hilf- und Hoffnungslosigkeit, Gefühle des Überwältigtseins oder Betäubtseins [Znoj, 2016]. 


\section{Psychologische und psychotherapeutische Interventionen}

\subsection{Modell palliativpsychologischen Arbeitens}

Berthold und Gramm [2019] beschreiben ein Modell, mit dem die palliativpsychologische Arbeit mit Patienten und Angehörigen durch fünf Interventionsfelder beschrieben werden kann (siehe auch Abb. 1).

1. In der psychologischen Beratung erfolgen Aufklärung und Information.

2. Die psychologische (Prozess-)Begleitung umfasst die drei Themenkomplexe Bewältigung, Entscheidung und Vermittlung.

3. Eine psychologische Behandlung kann als Erweiterung des Themenkomplexes Bewältigung bei Vorliegen subdiagnostischer Befunde notwendig werden. Eine psychotherapeutische oder psychiatrische (Mit-) Behandlung wird notwendig bei Vorliegen einer psychischen Störung.

4. Die psychologisch-ethische Klärung erweitert den Themenkomplex Entscheidung bei Vorliegen eines normativen Konflikts, indem intra- und interpsychische Aspekte der Entscheidungsfindung (etwa Schuldgefühle, Familien- oder Teamdynamik) explizit gemacht und geklärt werden. In dieser Funktion dient die psychologisch-ethische Klärung als Vorstufe der klinischen Ethikberatung.

5. Die psychologische Mediation kann als Erweiterung des Themenkomplexes Vermittlung bei tiefgreifenden Konflikten notwendig werden.

\subsection{Transfer Psychotherapeutischer Interventionen}

Sofern psychotherapeutische Interventionen zum Einsatz kommen, zielen diese selten auf die psychotherapeutische Behandlung psychopathologischer Störungen ab. In den meisten Fällen steht die Reduktion belastender psychischer sowie somatischer Symptome mit psychogenem Anteil aufgrund der palliativen Erkrankung im Vordergrund [Mehnert, 2015; Münch et al., 2015].

Das Potenzial psychotherapeutischer Ansätze wird am Lebensende jedoch insofern bedeutsam, als deren Strategien und Techniken, aber auch Konzepte und Grundhaltungen in übertragener und angepasster Form wirksam zum Einsatz kommen können [Berthold et al., 2017]. Im Kontext der Palliativversorgung kann daher von einem Transfer psychotherapeutischer Interventionen ausgegangen werden.

Hervorgehoben seien an dieser Stelle die systemischfamilientherapeutischen Ansätze, aus deren Repertoire das Erstellen eines Genogramms zur Erfassung des Familiensystems [Deutsche Gesellschaft für Palliativmedizin, 2016] sowie die Durchführung von Familiengesprächen zur Etablierung des Themas "Sterben", zum Abgleich unterschiedlicher Bedürfnislagen, zur Mediation bei Kon-

\section{Interventionsfelder der Palliativpsychologie}

Berthold \& Gramm, 2019
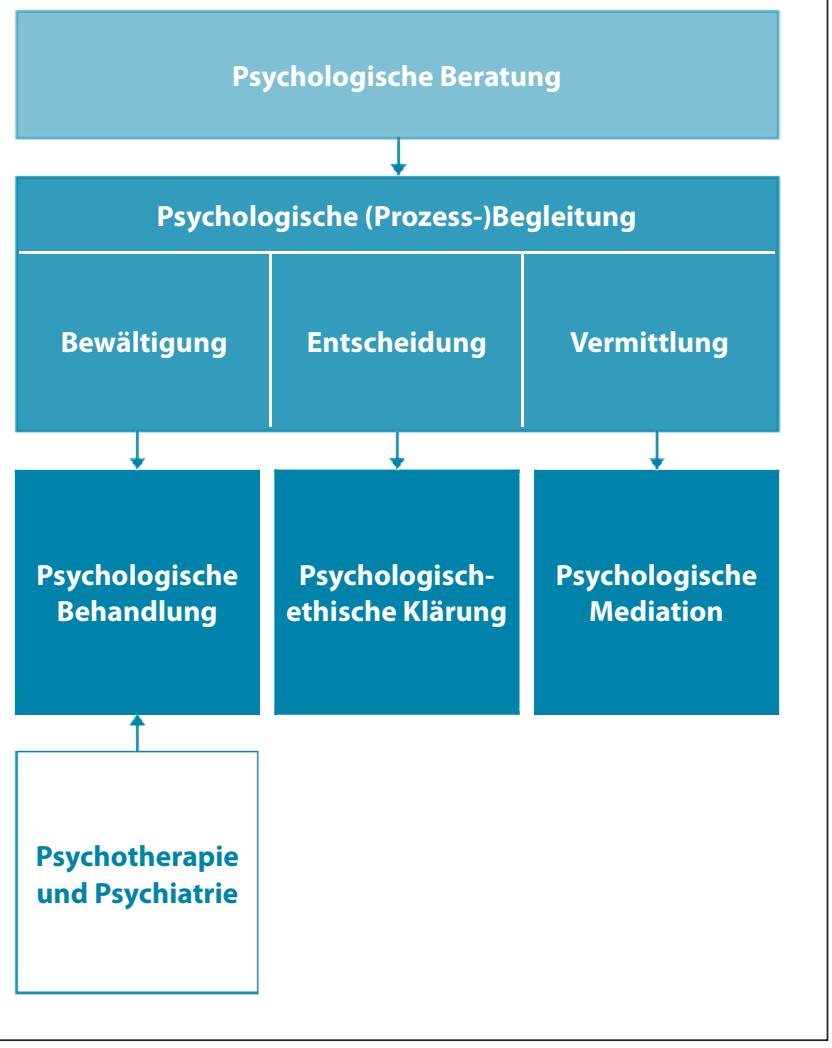

Fig. 1. Interventionsfelder der palliativpsychologischen Arbeit mit Patienten und Angehörigen.

flikten oder zur weiteren Behandlungs- und Versorgungsplanung für die psychologische (Prozess-)Begleitung und Behandlung sehr hilfreich sein können.

Vor diesem Hintergrund hoch ausgeprägter Stressniveaus kommen Entspannungsverfahren und Imaginationstechniken häufig zum Einsatz. Darüber hinaus verspricht auch die Anwendung von Hypnotherapie zur Symptomkontrolle bei Schmerzen, Atemnot, Übelkeit, Erbrechen, Appetitlosigkeit, Juckreiz oder Schlaflosigkeit wie auch zur psychosozialen und spirituellen Begleitung gute Erfolge [Schulze, 2013].

Insbesondere auch achtsamkeitsbasierte Ansätze erfahren in Palliative Care eine besondere Relevanz, da Patienten, die immer wieder "an die Grenzen von Machbarem, Planbarem stoßen", darin unterstützt werden können, "die Aufmerksamkeit auf die Realisierung und Veränderung innerer Erlebensprozesse" zu richten [Schulz-Kindermann et al., 2013, S. 148].

Ebenso können auch existenziell-humanistische Ansätze sinnvoll zur Anwendung kommen, insofern existenzielle Themen Patienten nicht nur stark beschäftigen, sondern auch erheblich belasten und existenzielle Ängste 
auslösen [Trachsel und Noyon, 2017]. Es eignen sich hier Methoden aus der existenziell-humanistischen Psychotherapie, wie sie auf der Basis der kontinentalen Existenzphilosophie und der angelsächsischen humanistischen Psychologie durch Vertreter wie James Bugenthal, Rollo May oder Irvin D. Yalom entwickelt wurde [Schneider und Krug, 2010].

\subsection{Spezifische Interventionen für die Arbeit mit Palliativpatienten}

4.3.1 Meaning-Centered Psychotherapy sinnzentrierte Psychotherapie

Eine Form existenzieller Psychotherapie, die im Vergleich zur oben beschriebenen existenziell-humanistischen Psychotherapie auf ein spezifisches existenzielles Thema fokussiert, ist die sinnzentrierte Psychotherapie [Breitbart et al., 2012]. Auf Grundlage der Logotherapie Viktor Frankls entwickelte Breitbart Interventionen, die darauf abzielen, Sinn und Bedeutung im Angesicht des Todes zu finden. Frankl hatte erkannt, "dass wir nicht mehr einfach nach dem Sinn des Lebens fragen, sondern dass wir uns selbst als die Befragten erleben, als diejenigen, an die das Leben täglich und stündlich Fragen stellt" [Frankl, 2008, S. 125]. Durch diese Umkehrung der Perspektive wird der Mensch auch in widrigen Umständen wieder zum Akteur, denn "Leben heißt letztlich eben nichts anderes als: Verantwortung tragen für die rechte Beantwortung der Lebensfragen, für die Erfüllung der Aufgaben, die jedem einzelnen das Leben stellt" [Frankl, 2008, S. 125]. Das Potenzial dieses therapeutischen Ansatzes für Menschen, die sich einer existenziell bedrohlichen Situation ausgesetzt sehen, ist offensichtlich.

Die Meaning-Centered Group Psychotherapy (MCGP) bietet einen Rahmen, in dem sich (onkologische) Patienten gemeinsam mit Fragen um Sinnhaftigkeit, inneren Frieden und Zielorientierung in ihrem Leben vor dem Hintergrund ihrer Erkrankung auseinandersetzen. In acht Sitzungen werden über Psychoedukation, Gruppendiskussion und Übungen unterschiedliche Schwerpunktthemen bearbeitet: 1. SinnKonzepte und Sinn-Quellen; 2. Krebs und Sinn; 3. Sinnschöpfung aus der Lebensgeschichte; 4. Sinnschöpfung aus der Gegenwart und Zukunft; 5. Sinnschöpfung aus Einstellungen (den Grenzen des Lebens begegnen); 6. Sinnschöpfung aus kreativem Tun und Verantwortungsübernahme; 7. Erlebnisbasierte Sinnschöpfung (Natur, Kunst, Humor); 8. Abschlusssitzung [Breitbart et al., 2004, 2010].

Da sich die Durchführung von Gruppensitzungen mit Palliativpatienten als schwierig erwies, wurde die Individual Meaning-Centered Psychotherapy (IMCP) entwickelt [Breitbart et al., 2012, 2018]. In sieben Sitzungen werden die Themenbereiche der MCGP im Einzelsetting erarbeitet.

\subsubsection{Kognitiv-Existenzielle Gruppen-}

Psychotherapie

Eine weitere Form existenzieller Psychotherapie für den Palliative Care-Kontext ist die kognitiv-existenzielle Gruppen-Psychotherapie, die primär für Patientinnen mit Brustkrebs entwickelt wurde [z.B. Kissane et al., 2003]. Dabei handelt es sich um eine manualisierte Gruppentherapie für sechs bis acht Patienten über 20 wöchentliche Sitzungen, die durch zwei Psychotherapeutinnen (mindestens eine Frau) geleitet wird und folgende sechs Ziele verfolgt: 1 . Herstellen einer supportiven Umgebung; 2 . Trauerarbeit unterstützen; 3 . Reframing von negativen Gedanken; 3. Einübung von Problemlöseverhalten und Bewältigungsstrategien; 4. Hoffnung vermitteln; und 6. Prioritäten für die (nahe) Zukunft setzen. Die Methode ist grundsätzlich auf Brustkrebspatientinnen zugeschnitten und in diesem Kontext empirisch am besten untersucht worden. Zentrale Themen der Gruppensitzungen beinhalten: "the threat of death; fear of recurrence; living with uncertainty; understanding treatment with chemotherapy, radiotherapy and hormones; the doctorpatient relationship; body- and self-image; sexuality; surgical reconstruction; relationship with partner, friends and family; and life style and future goals" [Kissane et al., 2003, S. 535]. Der Ansatz kann jedoch auf andere Patientengruppen übertragen werden.

\subsubsection{Dignity Therapy - würdezentrierte Therapie}

Da bei Palliativpatienten einer der am häufigsten genannten Gründe für den Wunsch nach vorzeitigem Sterben der Verlust der Würde ist, hat eine Arbeitsgruppe um Harvey Max Chochinov ein empirisch fundiertes Würde-Modell erstellt, das in drei Bereiche untergliedert ist: 1. Krankheitsbezogene Aspekte, 2. soziale Aspekte und 3. psychische Aspekte (würdebewahrendes Repertoire) [Chochinov, 2002]. Um die psychischen Aspekte zu stärken, wurde die würdezentrierte Therapie entwickelt [Chochinov et al., 2005]. Auf Grundlage eines Fragenkatalogs wird mit dem Patienten ein Interview geführt, in dem einerseits die Essenz der Persönlichkeit zum Ausdruck kommt (z.B. "Wann haben Sie sich am lebendigsten gefühlt?"), andererseits Wünsche und Anliegen für die Angehörigen festgehalten werden ("Was haben Sie über das Leben gelernt, das Sie gerne an andere weitergeben möchten?"). Das auf Band aufgenommene Gespräch wird transkribiert, editiert, dem Patienten vorgelesen und gegebenenfalls modifiziert. Nach Fertigstellung erhält der Patient ein Dokument, das er dann seinen Angehörigen weitergeben beziehungsweise hinterlassen kann. Neben dem ressourcenorientierten Blick auf die eigene Person stellt somit die Generativität einen weiteren wichtigen Wirkfaktor dar. Der Therapeut nimmt insofern eine systemische Position ein, als er nicht nur den Erzähler, sondern auch die Adressaten des 
Dokuments im Blick haben muss. Die würdezentrierte Therapie wurde auf unterschiedliche Anwendungsgebiete adaptiert und vielfach wissenschaftlich evaluiert [Martínez et al., 2017]. Mittlerweile liegt eine ausführliche Anleitung in Buchform auch auf Deutsch vor [Chochinov, 2017].

\subsubsection{Managing Cancer and Living Meaningfully}

Managing Cancer and Living Meaningfully (CALM) ist eine von der Forschergruppe um Dr. Gary Rodin entwickelte manualisierte, semi-strukturierte individuelle Kurzpsychotherapie für Menschen mit fortgeschrittener Erkrankung zur Reduktion von Stress und Förderung persönlichen Wachstums [Lo et al., 2014]. Durch therapeutische Unterstützung in Affektregulation, Bindungssicherheit und Mentalisierung wird auf vier Bereiche fokussiert: 1. Symptomlinderung und Kommunikation mit den Behandlern; 2. Veränderungen der eigenen Person und Beziehung zu nahen Angehörigen; 3. Lebenssinn; 4. Zukunft und Sterben. CALM vermittelt Patienten Sicherheit, um sich mit Ängsten konfrontieren zu können, um in ihrer Verletzlichkeit gesehen zu werden und um sich den Herausforderungen der fortschreitenden Erkrankung stellen zu können.

\subsection{Spezifische Interventionen für die Arbeit mit Angehörigen von Palliativpatienten}

Die Bedürfnisse und Belastungen von Angehörigen, und dementsprechend auch deren therapeutische Themen, unterscheiden sich teils erheblich von denen der Patienten selbst. Daher wurden auch für diese Klientengruppe Interventionsansätze entwickelt, die meist auch pflegerische und finanzielle Aspekte beinhalten und für das Gruppensetting konzipiert wurden. Interventionen, die sich an die Palliativversorgung bei Vorliegen einer komplizierten Trauer anschließen, werden an dieser Stelle nicht eigens angeführt.

\subsubsection{Kurzzeit-Gruppe für Angehörige}

In der Kurzzeit-Gruppe für Angehörige [Harding et al., 2002] wird in sechs Gruppensitzungen eine Kombination patientenbezogener Psychoedukation mit Austausch und sozialer Unterstützung inklusive Informationen zu finanzieller Unterstützung, Pflege, Aromatherapie oder Anleitung zur Selbstfürsorge angeboten.

\subsubsection{Training für pflegende Angehörige}

Beim Training für pflegende Angehörige (Caregiving at Life's End) [Kwak et al., 2007] wird in fünf Sitzungen der Austausch über Erfahrungen mit Pflege, Informationen zu praktischen Angelegenheiten (Finanzen, Recht, Beruf) und Förderung von Akzeptanz der Endlichkeit des Lebens, Sinn und Trauer besprochen.
4.4.3 Unterstützungsprogramm für Angehörige

Beim Unterstützungsprogramm für Angehörige [Henriksson und Andershed, 2007] werden in sechs Sitzungen allgemeine Informationen zu Palliative Care, Ernährung, praktischen Angelegenheiten, Krisenmanagement, Physio- und Ergotherapie vermittelt sowie existenzielle Fragen und eigene Ressourcen angesprochen.

4.4.4 Psychoedukationale Intervention für pflegende Angehörige

Bei der Psychoedukationalen Intervention für pflegende Angehörige [Hudson et al., 2005] werden die Angehörigen über ein Handbuch, Hausbesuche und Telefonate in der Informationssuche unterstützt und zu Selbstfürsorge und Sinnfindung angeleitet.

\subsubsection{Existenziell behaviorale Therapie für}

Angehörige

Die existenziell behaviorale Therapie für Angehörige (EBT) [Fegg et al., 2013] ist eine Verbindung von achtsamkeitsbasierter Verhaltenstherapie mit existenziell orientierter Psychotherapie fokussiert auf Achtsamkeit und Akzeptanz, Orientierung an Werten sowie Quellen von Lebenssinn und Ressourcenstärkung [Fegg et al., 2013; Fegg, 2016]. In sechs Gruppensitzungen werden psychoedukative Elemente, Gruppendiskussionen und erlebnisorientierte Ansätze kombiniert: 1. Kennenlernen, Einführung in Achtsamkeit; 2. Tod, Trauer und Achtsamkeit; 3. Ressourcenaktivierung und Sinnfindung; 4. Selbstfürsorge und Stressbewältigungsstrategien; 5. Persönliche Werte zur (Neu-)Orientierung; 6. Abschied und neue Schritte. Zusätzlich erhalten die Angehörigen eine $\mathrm{CD}$ mit Achtsamkeitsübungen zur täglichen Praxis.

\section{Ausblick}

Das Spektrum psychologischer und psychotherapeutischer Ansätze leistet einen wertvollen Beitrag für die Steigerung der Lebensqualität von Patienten und Angehörigen in Palliative Care. Auch wenn sich entsprechende Strukturen für eine bedarfsgerechte Versorgung von Palliativpatienten in Deutschland bislang noch nicht etabliert haben, so ist dennoch ein deutlicher Trend in diese Richtung festzustellen. Dabei muss die Verankerung psychologischer und psychotherapeutischer Arbeit in Palliative Care nicht nur durch die Einbindung von Psychotherapeuten in Palliative Care-Teams erfolgen. Niedergelassene Psychotherapeuten können eigeninitiativ zu Mitgliedern palliativer Netzwerke werden, indem sie beispielsweise Qualitätszirkel besuchen, Kooperationen mit ambulanten Palliative Care-Teams und mit Kliniken an- 
bieten oder sich ehrenamtlich in Ethikkomitees engagieren.

"Regardless of whether one works as a practitioner, an educator, or a clinical researcher designing and evaluating treatments, the field of palliative care offers unparalleled opportunities to change lives for the better. Moreover, by helping seriously ill patients and their families find connection and healing in the midst of medical suffering, we ourselves are privileged to find deeper meaning in our own lives through these powerful and inspiring human encounters" [Kasl-Godley et al., 2014, S. 372].

\section{Statement of Ethics}

Im Zusammenhang mit dieser Arbeit bestehen keine ethischen Konflikte.

\section{Disclosure Statement}

Die Autoren erklären hiermit, dass keine Interessenskonflikte bezüglich des Manuskriptes bestehen.

\section{Literatur}

Andrade C. Ketamine for Depression, 4: In What Dose, at What Rate, by What Route, for How Long, and at What Frequency? J Clin Psychiatry. 2017 Jul;78(7):e852-7.

Bausewein C. Bislang unterschätzt. Palliativmedizin für Nicht-Tumorpatienten. Klinikarzt. 2005;34(01/02):19-23.

Becker G, Blum HE. "Medical Futility”: Der Arzt im Spannungsfeld von Behandlungsauftrag und Behandlungsbegrenzung. Dtsch Med Wochenschr. 2004 Jul;129(31-32):1694-7.

Berthold D, Gramm J, Gaspar M, Sibelius U, editors. Psychotherapeutische Perspektiven am Lebensende. Göttingen: Vandenhoeck \& Ruprecht; 2017.

Berthold D, Gramm J. Transferarbeit: Psychotherapeutische Interventionen am Lebensende. PiD - Psychother Dialog. 2019; 20(01):32-6.

Berufsverband Deutscher Psychologinnen und Psychologen (BDP), editor. Berufsbild Psychologie. Psychologische Tätigkeitsfelder [Internet]. 2016 [cited 2018 Aug 1]. Available from: https://www.bdp-verband.de/bdp/archiv/berufsbild-psychologie.pdf

Block SD. Psychological issues in end-of-life care. J Palliat Med. 2006 Jun;9(3):751-72.

Block SD; ACP-ASIM End-of-Life Care Consensus Panel. American College of Physicians American Society of Internal Medicine. Assessing and managing depression in the terminally ill patient. Ann Intern Med. $2000 \mathrm{Feb}$; 132(3):209-18.

Breitbart W, Gibson C, Poppito SR, Berg A. Psychotherapeutic interventions at the end of life: a focus on meaning and spirituality. Can J Psychiatry. 2004 Jun;49(6):366-72.

Breitbart W, Pessin H, Rosenfeld B, Applebaum AJ, Lichtenthal WG, Li Y, et al. Individual meaning-centered psychotherapy for the treatment of psychological and existential distress: A randomized controlled trial in patients with advanced cancer. Cancer. 2018 Aug;124(15):3231-9.

Breitbart W, Poppito S, Rosenfeld B, Vickers AJ, Li Y, Abbey J, et al. Pilot randomized controlled trial of individual meaning-centered psychotherapy for patients with advanced cancer. J Clin Oncol. 2012 Apr;30(12):13049.

Breitbart W, Rosenfeld B, Gibson C, Pessin H, Poppito S, Nelson C, et al. Meaning-centered group psychotherapy for patients with advanced cancer: a pilot randomized controlled trial. Psychooncology. 2010 Jan;19(1):21-8.
Chochinov HM, Hack T, Hassard T, Kristjanson LJ, McClement S, Harlos M. Dignity therapy: a novel psychotherapeutic intervention for patients near the end of life. J Clin Oncol. 2005 Aug;23(24):5520-5.

Chochinov HM. Dignity-conserving care-a new model for palliative care: helping the patient feel valued. JAMA. 2002 May;287(17):225360

Chochinov HM. Würdezentrierte Therapie: was bleibt - Erinnerungen am Ende des Lebens. Göttingen, Bristol, CT: Vandenhoeck \& Ruprecht; 2017.

Clarke DM, Kissane DW. Demoralization: its phenomenology and importance. Aust N Z J Psychiatry. 2002 Dec;36(6):733-42.

Deutsche Gesellschaft für Palliativmedizin. Palliativmedizinisches Basisassessment (PBA) [Internet]. 2016 [cited 2019 Sep 3]. Available from: https://www.dgpalliativmedizin.de/images/Palliativmedizinisches_Basisassessment_2016_überarb_2018.pdf

Endicott J. Measurement of depression in patients with cancer. Cancer. 1984 May;53(10 Suppl): 2243-9.

Fegg M. Zusammenfassende Ergebnisse zur Existenziell Behavioralen Therapie (EBT) für Angehörige von Palliativpatienten. Palliativmedizin. 2016;17(02):76-82.

Fegg MJ, Brandstätter M, Kögler M, Hauke G, Rechenberg-Winter P, Fensterer V, et al. Existential behavioural therapy for informal caregivers of palliative patients: a randomised controlled trial. Psychooncology. 2013 Sep; 22(9):2079-86

Ferris FD, Balfour HM, Bowen K, Farley J, Hardwick $M$, Lamontagne $C$, et al. A model to guide patient and family care: based on nationally accepted principles and norms of practice. J Pain Symptom Manage. 2002 Aug; 24(2):106-23.

Frankl VE. ... trotzdem Ja zum Leben sagen: Ein Psychologe erlebt das Konzentrationslager. 29. Aufl., ungekürzte Ausg. München: Deutscher Taschenbuch-Verlag; 2008.

Grande G, Rowland C, van den Berg B, Hanratty B. Psychological morbidity and general health among family caregivers during end-of-life cancer care: A retrospective census survey. Palliat Med. 2018 Dec;32(10):1605-14.

Harding R, Leam C, Pearce A, Taylor E, Higginson IJ. A multi-professional short-term group intervention for informal caregivers of patients using a home palliative care service. J Palliat Care. 2002;18(4):275-81.
Heidenreich T, Michalak J, editors. Die “dritte Welle" der Verhaltenstherapie: Grundlagen und Praxis. 1st ed. Weinheim, Basel: Beltz; 2013.

Heidenreich T, Michalak J, Eifert G. Balance von Veränderung und achtsamer Akzeptanz: Die dritte Welle der Verhaltenstherapie. Psychother Psych Med. 2007;57(12):475-86.

Henriksson A, Andershed B. A support group programme for relatives during the late palliative phase. Int J Palliat Nurs. 2007 Apr; 13(4):175-83.

Hudson PL, Aranda S, Hayman-White K. A psycho-educational intervention for family caregivers of patients receiving palliative care: a randomized controlled trial. J Pain Symptom Manage. 2005 Oct;30(4):329-41.

Kasl-Godley JE, King DA, Quill TE. Opportunities for psychologists in palliative care: working with patients and families across the disease continuum. Am Psychol. 2014 May-Jun; 69(4):364-76.

Kern M, Grammatico D, Ostgathe E. "Was brauchen Menschen, die ihre schwerstkranken und sterbenden Angehörigen zu Hause versorgen?" - Ein Projekt von ALPHA Rheinland mit Unterstützung des Ministeriums für Arbeit, Gesundheit und Soziales des Landes Nordrhein-Westfalen [Internet]. Bonn: ALPHA-Rheinland; 2009 [cited 2017 Dec 10]. Available from: https://alpha-nrw.de/wpcontent/uploads/2014/07/was-brauchenmenschen.pdf

Kissane DW, Bloch S, Smith GC, Miach P, Clarke DM, Ikin J, et al. Cognitive-existential group psychotherapy for women with primary breast cancer: a randomised controlled trial. Psychooncology. 2003 Sep;12(6):532-46.

Kissane DW, McKenzie M, Bloch S, Moskowitz C, McKenzie DP, O’Neill I. Family focused grief therapy: a randomized, controlled trial in palliative care and bereavement. Am J Psychiatry. 2006 Jul;163(7):1208-18.

Kröger F, Altmeyer S, Hendrischke A. Systemische Familienmedizin. KONTEXT. 2002; 33(4):267-87.

Kwak J, Salmon JR, Acquaviva KD, Brandt K, Egan KA. Benefits of training family caregivers on experiences of closure during end-oflife care. J Pain Symptom Manage. 2007 Apr; 33(4):434-45. 
Leitlinienprogramm Onkologie (Deutsche Krebsgesellschaft, Deutsche Krebshilfe, AWMF), editor. Palliativmedizin für Patienten mit einer nicht heilbaren Krebserkrankung, Langversion 1.0 [Internet]. 2015 [cited 2018 Aug 1]. Available from: http://leitlinienprogramm-onkologie.de/Palliativmedizin. 80.0.html

Lo C, Hales S, Jung J, Chiu A, Panday T, Rydall A, et al. Managing Cancer And Living Meaningfully (CALM): phase 2 trial of a brief individual psychotherapy for patients with advanced cancer. Palliat Med. 2014 Mar;28(3):234-42.

Martínez M, Arantzamendi M, Belar A, Carrasco JM, Carvajal A, Rullán M, et al. 'Dignity therapy', a promising intervention in palliative care: A comprehensive systematic literature review. Palliat Med. 2017 Jun;31(6):492-509.

Maugham WS. Sheppey. London: Heinemann; 1933.

Mehnert A, Vehling S, Höcker A, Lehmann C, Koch U. Demoralization and depression in patients with advanced cancer: validation of the German version of the demoralization scale. J Pain Symptom Manage. 2011 Nov; 42(5):768-76.

Mehnert A. Psychotherapie in der palliativen Versorgung. Psychother Psychosom Med Psychol. 2015 Sep;65(9-10):387-96.

Meran JG. Lebensqualität, Aussichtslosigkeit und Therapiebegrenzung. Imago Hominis. 2003; 10:19-28.

Miller K, Massie MJ. Depressive Disorders. In: Holland JC, Breitbart WS, Jacobsen PB, Loscalzo MJ, McCorkle R, Butow PN, editors. Psycho-oncology. 3rd ed. Oxford, New York: Oxford University Press; 2015. pp. 311-8.

Mitchell AJ, Chan M, Bhatti H, Halton M, Grassi L, Johansen C, et al. Prevalence of depression, anxiety, and adjustment disorder in oncological, haematological, and palliative-care settings: a meta-analysis of 94 interview-based studies. Lancet Oncol. 2011 Feb;12(2):16074.

Monforte-Royo C, Villavicencio-Chávez C, Tomás-Sábado J, Mahtani-Chugani V, Balaguer A. What lies behind the wish to hasten death? A systematic review and meta-ethnography from the perspective of patients. PLoS One. 2012;7(5):e37117.
Münch U, Gramm J, Berthold D. Mehr als Psychotherapie. Psychologisches Arbeiten in Palliative Care. PiD - Psychother Dialog. 2016; 17(01):81-5.

Münch U, Gramm J, Jentschke E, Mai S. Palliativpsychologie - Ist eine Approbation wirklich notwendig? Palliativmedizin. 2015;16(05): 191-191.

Ohnsorge K, Gudat H, Rehmann-Sutter C. Intentions in wishes to die: analysis and a typology - A report of 30 qualitative case studies of terminally ill cancer patients in palliative care. Psychooncology. 2014 Sep;23(9):1021-6.

Preisler M, Goerling U. Angehörige von an Krebs erkrankten Menschen. Onkologe. 2016;22(5): $336-41$.

Prigerson HG, Bierhals AJ, Kasl SV, Reynolds CF 3rd, Shear MK, Newsom JT, et al. Complicated grief as a disorder distinct from bereavement-related depression and anxiety: a replication study. Am J Psychiatry. 1996 Nov; 153(11):1484-6.

Robinson S, Kissane DW, Brooker J, Burney S. A systematic review of the demoralization syndrome in individuals with progressive disease and cancer: a decade of research. J Pain Symptom Manage. 2015 Mar;49(3):595-610.

Röhrle B, Caspar F, Schlottke PF, editors. Lehrbuch der klinisch-psychologischen Diagnostik. 1st ed. Stuttgart: Kohlhammer; 2008.

Sauer T, Weiske K. Angewandte Ethik in der Intensivmedizin: Zwischen medizinischer Indikation und Patientenwille. In: Eckart J, Weigand MA, Briegel J, editors. Intensivmedizin (Kompendium und Repetitorium zur interdisziplinären Weiter- und Fortbildung). Landsberg: ecomed Medizin; im Druck.

Schneider KJ, Krug OT. Existential-humanistic therapy. 1st ed. Washington (DC): American Psychological Association; 2010.

Schneiderman LJ, Jecker NS, Jonsen AR. Medical futility: its meaning and ethical implications. Ann Intern Med. 1990 Jun;112(12):949-54.

Schulze W. Hypnose und Hypnotherapie in der Palliativmedizin - Symptombehandlung und spirituelle Begleitung. Palliativmedizin. 2013; 14(02):59-72.

Schulz-Kindermann F, Koch U, Berger H, Hoffmann P, Huse-Kleinstoll G, Lang K, et al. Psychoonkologie: Grundlagen und psychotherapeutische Praxis. 1st ed. Weinheim, Basel: Beltz; 2013.
Sektion Psychologie der DGP, editor. Palliativpsychologie - Berufsbild für Psychologinnen und Psychologen in der Palliativversorgung [Internet]. 2016 [cited 2017 Apr 18]. Available from: https://www.dgpalliativmedizin. de/images/Berufsbild_PalliativpsychologIn DGP_2016.pdf

Serfaty M, King M, Nazareth I, Moorey S, Aspden T, Tookman A, et al. Manualised cognitivebehavioural therapy in treating depression in advanced cancer: the CanTalk RCT. Health Technol Assess. 2019 May;23(19):1-106.

Strada EA. Grief, demoralization, and depression: diagnostic challenges and treatment modalities. Prim Psychiatry. 2009 May; 16:7.

Trachsel M, Hermann H, Biller-Andorno N. Urteilsfähigkeit: Ethische Relevanz, konzeptuelle Herausforderung und ärztliche Beurteilung. Schweiz Med Forum. 2014a;14(11): 221-225.

Trachsel M, Krones T, Wild V. Zwangsernährung oder Palliative Care bei chronischer Anorexia nervosa? Behandlungsstrategien aus medizinethischer Sicht. In: Moos T, RehmannSutter C, Schües C, editors. Randzonen des Willens. Frankfurt am Main: Peter Lang; 2016. pp. 173-88.

Trachsel M, Maercker A. Lebensende, Sterben und Tod. 1st ed. Göttingen: Hogrefe; 2016.

Trachsel M, Mitchell C, Biller-Andorno N. Advance directives between respect for patient autonomy and paternalism. In: Lack P, BillerAndorno N, Brauer S, editors. Advance Directives. Dordrecht: Springer Netherlands; 2014b. pp. 169-79.

Trachsel M, Noyon A. Ratgeber Lebensende, Sterben und Tod: Informationen für Betroffene und Angehörige. 1st ed. Göttingen: Hogrefe; 2017.

Willig C, Wirth L. A meta-synthesis of studies of patients' experience of living with terminal cancer. Health Psychol. 2018 Mar;37(3):22837.

World Health Organization, editor. National cancer control programmes: policies and managerial guidelines. 2nd ed. Geneva: World Health Organization; 2002.

Znoj H. Komplizierte Trauer. 2., überarbeitete Auflage. Göttingen: Hogrefe; 2016. 